\title{
Experiencias
}

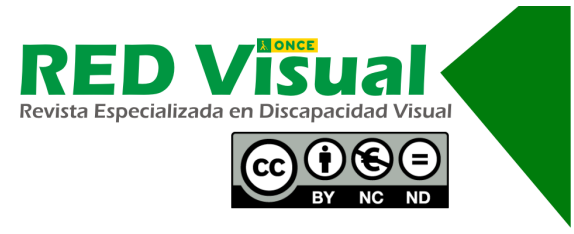

\section{Estudio radiológico de cadera mediante percepción táctil dirigido a estudiantes de Fisioterapia con discapacidad visual ${ }^{1}$}

\author{
Tactile perception in the radiological study of the hip \\ for physical therapy students with visual disability
}

M. ${ }^{a}$ P. Martín Rubio, L.F. Arribas González

\section{Resumen}

La luxación de cadera constituye la segunda causa de trastorno ortopédico en niños con parálisis cerebral. El mejor tratamiento de la misma es la prevención, donde la fisioterapia, a través de los programas de educación motriz terapéutica juega un papel fundamental, y, para ello, es necesario conocer la evolución del desarrollo de la cadera mediante protocolos de evaluación clínica y radiológica. Dado que la radiografía es una prueba diagnóstica que requiere de capacidad visual para su valoración e interpretación, hemos visto la necesidad de desarrollar una herramienta táctil para que nuestros alumnos adquieran estos conocimientos y sean profesionales altamente cualificados. Este proyecto tiene como objetivo ayudar a los estudiantes de Fisioterapia con discapacidad visual a interpretar mediante el tacto la disposición de los elementos óseos, tal y como aparecen en una radiografía de la cadera normal, y de la patológica en los niños afectados con parálisis cerebral, además de conocer los ángulos y las líneas necesarios para estudiar el estado ortopédico de la cadera. Está formado por tres series de láminas táctiles que representan una cadera normal, una cadera con signos patológicos leves y otra con signos patológicos graves, extraídas de radiografías de casos clínicos reales. Esta propuesta educativa es de igual utilidad para el docente, ya que facilita la transmisión con claridad de la complejidad de los conceptos. Se valoraron los resultados del

1 Trabajo galardonado con el Primer Premio en el IV Concurso de Experiencias de Innovación y Buenas Prácticas en Servicios Sociales de la ONCE, presentado con el título Estudio radiológico de cadera mediante percepción táctil.

Martín, M.P., y Arribas, L.F. (2020). Estudio radiológico de cadera mediante percepción táctil dirigido a estudiantes de Fisioterapia con discapacidad visual. RED Visual: Revista Especializada en Discapacidad Visual, 76, 11-23. https://doi.org/10.53094/CQIR4487. 
proyecto mediante una encuesta que fue cumplimentada por los alumnos que habían cursado la asignatura en el año académico anterior. El $100 \%$ de los alumnos manifestó que el nuevo material docente facilitaba la adquisición de los conocimientos.

\title{
Palabras clave
}

Fisioterapia. Parálisis cerebral. Radiografía de caderas. Discapacidad visual. Percepción táctil.

\begin{abstract}
Hip dislocation is the second most frequent cause of orthopaedic disorder in children with cerebral palsy. The best treatment is prevention, in which the physical therapist, deploying therapeutic motor education programmes, plays an essential role. Success in that role depends on monitoring hip development further to clinical and radiological assessment protocols. Inasmuch as assessing and interpreting X-rays calls for visual capacity, a tactile tool had to be developed for visually impaired physical therapy students to enable them to acquire the skills demanded of highly qualified professionals. The aim of this project was to help physical therapy students with visual disability interpret, by touch, the positions of the elements both in a healthy hipbone and in the pathological hips of children with cerebral palsy, in addition to understanding the angles and lines required to study orthopaedic status. The teaching material consists in three series of tactile lamina drawn from actual clinical X-rays: one representing a healthy hip, the second a hip with mild and the third with severe pathologies. This educational proposal is also useful for professors, for it enhances the clarity of the explanation of complex concepts. Project results were assessed with a questionnaire distributed among students who had taken the course the year before. All $(100 \%)$ of the respondents claimed that the new teaching material facilitated acquisition of the respective know-how.
\end{abstract}

\section{Key words}

Physical therapy. Cerebral palsy. Hip X-rays. Visual impairment. Tactile perception.

\section{Presentación y justificación}

Este proyecto se ha desarrollado en el marco de la asignatura de Fisioterapia en Afecciones Neurológicas, perteneciente al tercer curso del Grado en Fisioterapia de la

Martín, M.P., y Arribas, L.F. (2020). Estudio radiológico de cadera mediante percepción táctil dirigido a estudiantes de Fisioterapia con discapacidad visual. RED Visual: Revista Especializada en Discapacidad Visual, 76, 11-23. https://doi.org/10.53094/CQIR4487. 
Escuela Universitaria de Fisioterapia de la ONCE, adscrita a la Universidad Autónoma de Madrid.

Son muchos los autores que coinciden en que el mejor tratamiento de la luxación de cadera es la prevención. Para alcanzar este objetivo, es necesario establecer programas de seguimiento basados en pruebas y técnicas de valoración clínica y por imagen, como la radiografía de pelvis o cadera.

La indicación de la radiografía anteroposterior de pelvis o radiografía de caderas está justificada para el diagnóstico y seguimiento de las caderas en la parálisis cerebral debido a su simplicidad, a la confiabilidad de sus parámetros y al beneficio económico frente a otras pruebas de diagnóstico por imagen (Fernández-Palazzi y Guillén, 2007). Así mismo, la radiografía es la modalidad de detección sistemática preferida para la displasia del desarrollo de la cadera a partir de los 6 meses de edad (Vitale y Skaggs, 2010).

La radiografía de caderas debe formar parte de los programas de vigilancia para la prevención de la subluxación o luxación completa de caderas, complicación dolorosa y grave presente en alrededor del $50 \%$ de los niños con parálisis cerebral espástica significativa no deambulantes, ya que han demostrado reducir la incidencia de luxación y la necesidad de cirugía de rescate. A todo niño con parálisis cerebral y afectación funcional importante se le debería hacer una radiografía de las caderas anualmente desde los 2 años de edad, y antes o con más frecuencia si hay sospecha de clínica de subluxación (Gordons y Simkiss, 2006). En este mismo sentido, Murray y Robb (2006) consideran oportuna la prescripción de la radiografía de caderas anualmente o cada 6 meses dependiendo de la gravedad del cuadro clínico.

El fisioterapeuta juega un papel importante en la reeducación motriz terapéutica y en el control ortopédico de las caderas en niños con parálisis cerebral, de aquí la importancia de la inclusión, dentro de los programas de formación del Grado en Fisioterapia, de los conceptos relativos a cuáles son los parámetros que se valoran mediante una radiografía, tanto en la cadera normal como en la patológica, que le ayuden a adquirir las competencias necesarias para el correcto ejercicio de su profesión en el ámbito clínico e investigador.

Por ser la radiografía la técnica por imagen de elección para el seguimiento y valoración de la cadera en niños, por la frecuencia de su prescripción, por la prevalencia

Martín, M.P., y Arribas, L.F. (2020). Estudio radiológico de cadera mediante percepción táctil dirigido a estudiantes de Fisioterapia con discapacidad visual. RED Visual: Revista Especializada en Discapacidad Visual, 76, 11-23. https://doi.org/10.53094/CQIR4487. 
de la subluxación o luxación de cadera en niños con parálisis cerebral, por ser el fisioterapeuta parte del equipo interdisciplinar para el seguimiento de la problemática de caderas en esta población, se justifica la necesidad de que los conceptos inherentes al estudio radiológico de la cadera normal y patológica sean entendidos con claridad por parte del estudiante de Fisioterapia. Así mismo, todos estos hechos justifican la necesidad de que la docente desarrolle este proyecto para intentar conseguir que el alumno estudiante de Fisioterapia o fisioterapeuta con discapacidad visual adquiera con claridad todos estos conceptos, complejos de asimilar y transmitir al no poder contar con las radiografías como medio de apoyo visual.

La formación del alumno con discapacidad visual en todas sus competencias profesionales le permitirá ser más competitivo en el mundo laboral.

Durante años, se ha puesto de manifiesto la dificultad tanto por parte de la docente de transmitir con claridad estos conceptos sin un apoyo con medios visuales, como de su comprensión por parte del alumno con discapacidad visual, lo que ha constituido un objeto continuo de modificación de materiales de apoyo hasta llegar al desarrollo del proyecto actual.

El proyecto consiste en tres series que comprenden tres láminas táctiles cada una de ellas. Las láminas de cada serie se encuentran estructuradas de la siguiente manera:

- Serie 1, dedicada al estudio de los signos que se deben observar en una radiografía de una cadera normal. Formada por tres láminas táctiles. La lámina 1.1 representa las estructuras óseas presentes en una radiografía de caderas normal, permite al alumno reconocer mediante el tacto su morfología y disposición. Esta información ha sido extraída de una radiografía de caderas normal de un niño. Utilizando esta lámina de base se ha construido otra, la lámina 1.2, en la que aparecen los huecos para encajar las distintas líneas y arcos que se deben trazar para valorar una radiografía de caderas y cuáles son los valores de normalidad. Una tercera lámina, lámina 1.3, representa la información completa, y se le ha insertado un código QR en la esquina superior izquierda que incluye toda la información para que el alumno la pueda utilizar como herramienta de repaso y estudio durante su tiempo de aprendizaje autónomo.

- Serie 2, destinada al estudio radiológico de los signos que se pueden observar en una cadera de un niño con patología leve. Comprende tres láminas táctiles.

Martín, M.P., y Arribas, L.F. (2020). Estudio radiológico de cadera mediante percepción táctil dirigido a estudiantes de Fisioterapia con discapacidad visual. RED Visual: Revista Especializada en Discapacidad Visual, 76, 11-23. https://doi.org/10.53094/CQIR4487. 
La lámina 2.1 representa los elementos óseos, su disposición y morfología extraídos de la radiografía con patología leve y que el alumno puede apreciar mediante el tacto. La lámina 2.2 contiene los huecos para insertar las líneas y arcos que se trazan para valorar la radiografía y cuáles son los valores de los parámetros objeto de estudio en caso de patología leve. Una tercera lámina, lámina 2.3, representa la información completa, y se le ha insertado un código QR en la esquina superior izquierda que incluye toda la información para que el alumno la puede utilizar como herramienta de repaso y estudio durante su tiempo de aprendizaje autónomo.

- Serie 3, destinada al estudio radiológico de los signos que se pueden observar en una cadera de un niño con patología grave. Al igual que las anteriores, comprende tres láminas táctiles $(3.1,3.2$ y 3.3$)$ con el mismo enfoque que las de la Serie 2 , pero en una radiografía con patología grave.

Como se especificará más adelante, cada estructura ósea se ha diseñado en una textura diferente y se han establecido una serie de colores para facilitar el aprendizaje y la memorización de los conceptos.

\section{Objetivos}

1. Facilitar a los estudiantes y profesionales de Fisioterapia la adquisición de conocimientos para que les permita:

- llevar a cabo un razonamiento crítico,

- establecer un diagnóstico en fisioterapia,

- implementar un plan de intervención en fisioterapia y evaluar los resultados del mismo,

- participar en la promoción de la salud y la prevención de la enfermedad.

2. Dar a los docentes que atienden alumnos con discapacidad visual una herramienta de trabajo para que facilite la transmisión del conocimiento y la adquisición de este por parte del alumno.

Martín, M.P., y Arribas, L.F. (2020). Estudio radiológico de cadera mediante percepción táctil dirigido a estudiantes de Fisioterapia con discapacidad visual. RED Visual: Revista Especializada en Discapacidad Visual, 76, 11-23. https://doi.org/10.53094/CQIR4487. 


\section{Población destinataria de la experiencia}

Este proyecto de innovación docente puede ser de interés a tres poblaciones diana:

1. Docentes: pretende facilitar la labor docente de los profesores que atiendan a alumnos con algún tipo de discapacidad visual y que deben adquirir los conocimientos relacionados con el tema objeto de este proyecto.

2. Estudiantes de Fisioterapia y/o de otras áreas de Ciencias de la Salud con discapacidad visual que incluyan dentro de sus planes de estudio estas competencias.

3. Profesionales fisioterapeutas y/o de otras áreas de Ciencias de la Salud con discapacidad visual que quieran profundizar sobre este tema y requieran de un material adaptado para su estudio.

\section{Temporalización y técnicas de realización}

El proyecto se inició en el curso académico 2018-2019 y ha finalizado en septiembre de 2019, acorde a la siguiente temporalización:

1. Noviembre de 2018: tras la explicación de los conceptos en clase y la dificultad para su comprensión por parte de una alumna, la profesora se plantea la posibilidad de mejorar el material de apoyo existente en el momento.

2. Entre los meses de noviembre de 2018 y febrero de 2019 se trabaja en el proyecto, realizando algunas mejoras en el material de apoyo, dando como resultado el primer diseño.

3. Febrero de 2019: se concierta una primera reunión con el equipo del Centro de Tiflotecnología e Innovación de la ONCE (CTI) para presentarles el primer proyecto y ver la viabilidad de su desarrollo.

4. De febrero a junio de 2019 se trabaja en la mejora del proyecto. Tras la reunión con el CTI y conocer cómo trabajan, los tipos de proyectos que desarrollan, los 
materiales y tecnologías con los que cuentan, se decide dar un cambio al proyecto para hacerlo más accesible a la persona con discapacidad visual. Durante este periodo se ejecutaron varias tareas:

- Selección de radiografías de caderas, tanto normales como patológicas, que pusieran de manifiesto tanto la normalidad como los signos patológicos más frecuentes.

- Selección de los colores del fondo de las láminas, de los elementos óseos y las líneas para facilitar la memorización por parte de los alumnos con resto visual funcional y que pudieran ser percibidos por la mayor parte de alumnos con discromatopsia. Lectura de artículos científicos sobre el tema y consulta a oftalmólogos especializados en deficiencia visual y técnicos de rehabilitación básica y visual de la ONCE.

- Reunión del equipo para ver cómo extraer de la radiografía de los casos clínicos un esquema para eliminar elementos que distorsionasen la comprensión; elección de los colores y adaptación del texto en braille, siguiendo las normas de los documentos técnicos vigentes publicados por la Comisión Braille Española (CBE).

- Se obtienen las láminas en papel y se muestran a diferentes compañeros con trastornos en la percepción de los colores para comprobar si perciben todos los elementos de las mismas y detectar posibles aspectos de mejora.

5. Julio de 2019: presentación del proyecto al equipo del CTI para su desarrollo.

6. Agosto de 2019: conversaciones telefónicas con el CTI para ver la evolución del proyecto; elaboración del presente documento y las encuestas para la valoración del mismo.

7. Septiembre de 2019: reunión con el equipo del CTI para estudiar el primer prototipo. Presentación del proyecto a los alumnos del curso 2019-2020 y valoración de las diferencias respecto al material didáctico empleado en la impartición de la clase magistral participativa del curso anterior. 


\section{Metodología}

A continuación, se describirá el proceso de elaboración de los diferentes elementos que forman parte del presente proyecto.

1. Selección de radiografías. Se hace un análisis retrospectivo de las radiografías que posee la docente sobre casos clínicos que han sido atendidos por ella. Se seleccionan tres radiografías que reflejan con claridad los conceptos que el docente quiere transmitir. Se eliminan los datos identificativos de las mismas y se solicita a los padres de los niños la oportuna autorización para utilizar las imágenes radiográficas de sus hijos para elaborar este proyecto.

2. Se extrae de cada una de las radiografías, por medio de programas específicos de diseño gráfico (Adobe Photoshop y Adobe Illustrator), los elementos óseos objeto de estudio respetando su morfología y disposición, y se crean las láminas 1.1 (radiografía de cadera normal; Figura 1), 2.1 (radiografía de cadera con patología leve; Figura 2) y 3.1 (radiografía de cadera con patología grave; Figura 3). Se eligen los colores del fondo de la lámina, así como de los elementos óseos, y se incluye la información en braille con la fuente y las normas especificadas por la CBE para facilitar al alumno la comprensión de las explicaciones de la profesora durante la clase magistral participativa. Se establecen texturas diferentes para cada estructura ósea. Al introducir color se pretende favorecer la memorización en aquellos alumnos con resto visual funcional. Para la elección de los colores se revisa información relacionada con el tema y se hace una consulta a la Dr.a López López, oftalmóloga pediátrica del Hospital 12 de Octubre de Madrid, especializada en deficiencia visual cerebral. Los colores elegidos son amarillo, azul, negro, blanco y naranja. Dentro de las estructuras coloreadas en blanco o negro se va a introducir trama (puntos) en el color contrario; es decir, dentro de los elementos coloreados en blanco se incluirán puntos de color negro y viceversa, de esta forma todas las estructuras negras o blancas podrán ser percibidas por todo el mundo. Sabemos que no es posible abarcar todas las discromatopsias con una sola lámina, pero, teniendo en cuenta que las personas con dificultades para percibir el rojo (protanopes) y el verde (deuteranopes) son más numerosas, se decide elegir colores azules-amarillos, ya que la prevalencia de personas con problemas para percibir dichos colores es bastante menor.

Martín, M.P., y Arribas, L.F. (2020). Estudio radiológico de cadera mediante percepción táctil dirigido a estudiantes de Fisioterapia con discapacidad visual. RED Visual: Revista Especializada en Discapacidad Visual, 76, 11-23. https://doi.org/10.53094/CQIR4487. 
3. Se elaboran las láminas 1.3 (Figura 1), 2.3 (Figura 2) y 3.3 (Figura 3) correspondientes a la radiografía de cadera normal, con patología leve y con patología grave, respectivamente. Estas láminas contienen la información completa, todas las estructuras óseas y las líneas y arcos necesarios para el estudio de los parámetros característicos de cada una de ellas. La información explicativa de estas láminas será incluida en los códigos QR dispuestos en la esquina superior izquierda de la lámina. Estas láminas se elaboran para el estudio del alumno durante su tiempo de aprendizaje autónomo.

Figura 1. Radiografía, lámina 1.1 y lámina 1.3 de una cadera normal

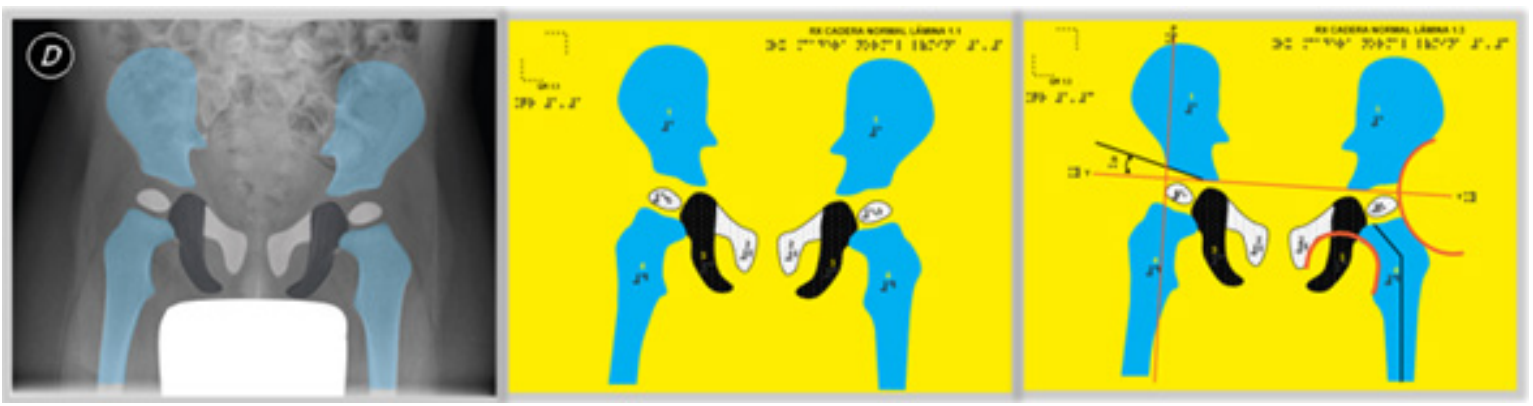

Figura 2. Radiografía, lámina 2.1 y lámina 2.3 de una cadera con patología leve

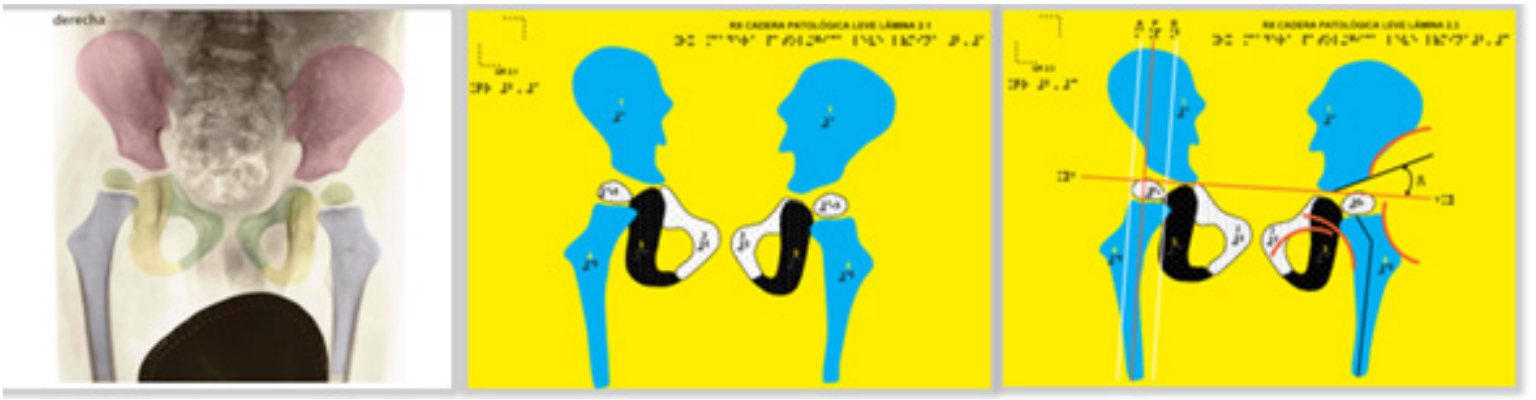

Figura 3. Radiografía, lámina 3.1 y lámina 3.3 de una cadera con patología grave

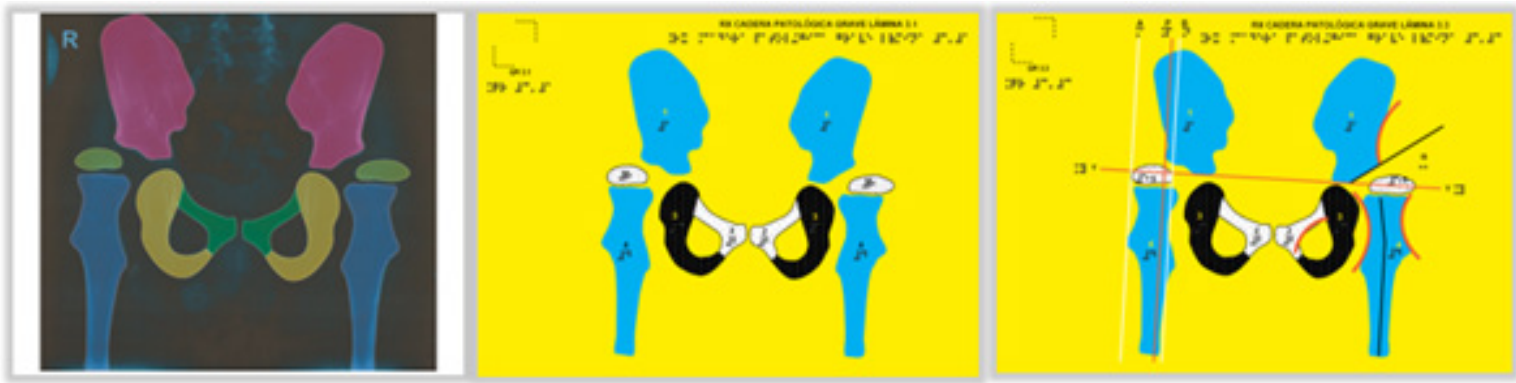

Martín, M. P., y Arribas, L.F. (2020). Estudio radiológico de cadera mediante percepción táctil dirigido a estudiantes de Fisioterapia con discapacidad visual. RED Visual: Revista Especializada en Discapacidad Visual, 76, 11-23. https://doi.org/10.53094/CQIR4487. 
4. Se acuerda con el equipo del CTI la fabricación de las láminas 1.2, 2.2 y 3.2 utilizando para ello las láminas 1.3, 2.3 y 3.3 dejando los huecos correspondientes a las líneas y arcos contenidas en ellas para que el alumno las pueda encajar durante la clase magistral participativa de la profesora (figura 4).

Figura 4. Imagen de la lámina 2.2 de una cadera con patología leve

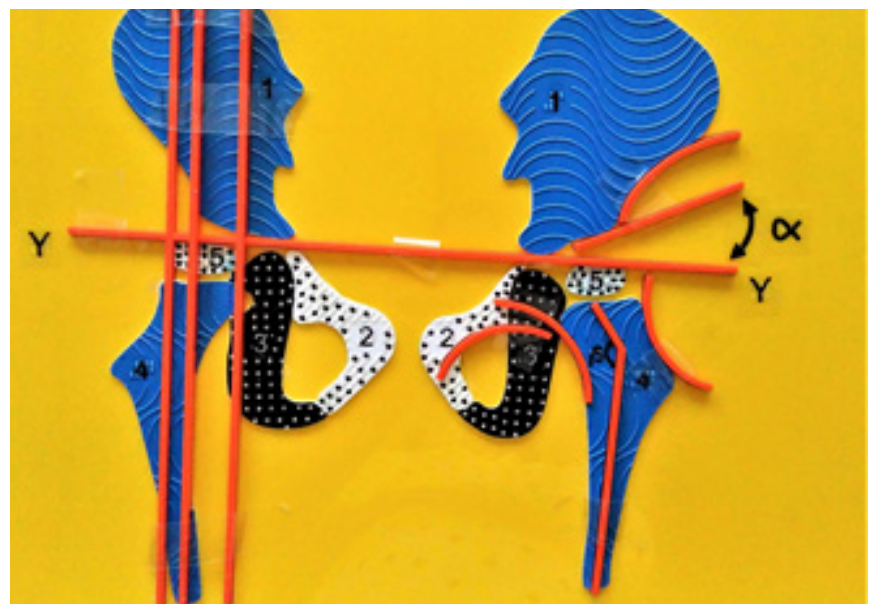

5. Toda la documentación relativa al proyecto es enviada por correo electrónico al CTI para la elaboración de cada una de las series. Además de lo anterior, se incluye un documento Word que contiene las líneas y arcos necesarios, para encajar en las láminas $1.2,2.2$ y 3.2 .

\section{Resultados}

Figura 5. Imagen de una clase magistral

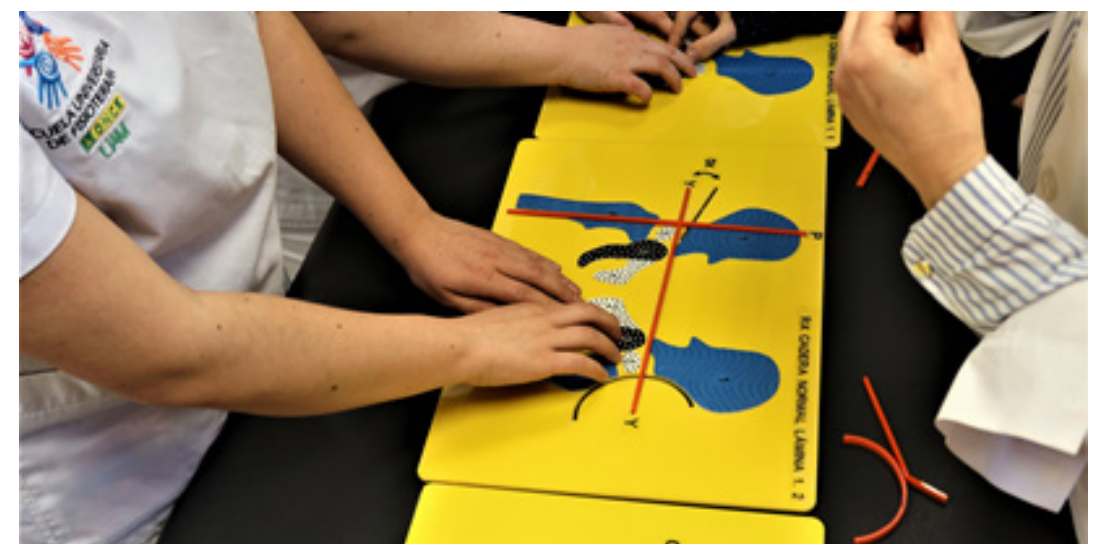

Martín, M.P., y Arribas, L.F. (2020). Estudio radiológico de cadera mediante percepción táctil dirigido a estudiantes de Fisioterapia con discapacidad visual. RED Visual: Revista Especializada en Discapacidad Visual, 76, 11-23. https://doi.org/10.53094/CQIR4487. 
El proyecto fue presentado a siete alumnos (Figura 5) que cursaron la asignatura en el curso académico anterior y adquirieron los conocimientos con otro material de apoyo. El objetivo que se perseguía era la valoración del mismo por parte del alumno y la detección de problemas para poder ser solventados. Con este fin, y tras presentarles el proyecto, se les pasó una encuesta de la que se extrajo la siguiente información en cada una de las preguntas.

El $100 \%$ de los alumnos cumplimentó la encuesta, obteniéndose los siguientes resultados para cada una de las preguntas realizadas:

1. ¿El material docente facilita la adquisición de conocimientos?

El 100\% de los alumnos contestó que sí. Uno de ellos hizo la siguiente apreciación: «Gracias al material, el aprendizaje es más rápido y sencillo, ya que al poderlo tocar tienes una imagen más clara».

2. Esta pregunta va destinada a las personas con dificultades en la percepción de los colores: ¿ha tenido dificultades para la identificación de alguno de los colores?

Dos de los alumnos no percibieron adecuadamente uno de los colores. Uno de ellos percibió el naranja como un amarillo más intenso que el tono del fondo de la lámina. Sugirió su sustitución por rojo para mejorar el contraste.

El otro alumno dudó si el color negro era tal o se trataba de marrón. No hizo ninguna sugerencia al respecto.

3. ¿Considera útil incluir las láminas 1.3, 2.3 y 3.3, con la información en códigos $Q R$, dentro del aula virtual como material de repaso para su proceso de autoaprendizaje?

4. El 100\% de los alumnos contestó que sí. Sus justificaciones al respecto fueron:

- «Me parece un material muy completo y útil para el alumno. Si tienes resto visual, te puedes aprovechar de él al ser imágenes más claras y grandes que lo que podemos apreciar en una radiografía debido a nuestra baja visión. Si no tienes resto visual, cuentas con la descripción detallada de la lámina».

Martín, M.P., y Arribas, L.F. (2020). Estudio radiológico de cadera mediante percepción táctil dirigido a estudiantes de Fisioterapia con discapacidad visual. RED Visual: Revista Especializada en Discapacidad Visual, 76, 11-23. https://doi.org/10.53094/CQIR4487. 
- «Es muy útil tener las láminas y la información en el aula virtual para poderla consultar en cualquier momento».

- «Es una manera más fácil de consultar la información impartida en clase sin tener que consultar al profesor».

\section{Conclusiones}

Tras el análisis de los resultados de las encuestas, creemos que este nuevo proyecto ayuda a los alumnos a comprender mejor todos los parámetros que deben ser valorados en una radiografía de caderas y sus repercusiones clínicas.

La posibilidad de recibir la información por dos sistemas sensoriales (visual y táctil) facilita la integración de los conocimientos.

Somos conscientes de que se ha valorado en un número muy reducido de alumnos, pero seguiremos llevándolo a la práctica para introducir, si fuera necesario, todas las mejoras oportunas para facilitar el aprendizaje de los alumnos con discapacidad visual y ayudarles a adquirir todas las competencias necesarias para desempeñar con mayor éxito su ejercicio profesional.

Respecto a la elección de colores para mejorar la percepción de los mismos, nos parece difícil abarcar todos los problemas, como se justificó anteriormente, pero nos centramos en que el alumno sea capaz de reconocer todos los elementos de la lámina, aunque el color que ellos perciban no se corresponda con el real.

\section{Referencias bibliográficas}

Fernández-Palazzi, F., y Guillén, A. J. (2007). Evaluación de la radiografía de cadera frente a la tomografía tridimensional en pacientes con parálisis cerebral. Revista de Ortopedia y Traumatología, 51(1), 30-34. (DOI: https://doi.org/10.1016/S0482-5985(07)74562-5).

Gordon, G. S. Simkiss, D. E. (2006). A systematic review of the evidence for hip surveillance in children with cerebral palsy [PDF]. The Journal of Bone and Joint Surgery, 88(B), 1492-1496. (DOI: https://doi.org/10.1302/0301-620x.88b11.18114).

Martín, M.P., y Arribas, L.F. (2020). Estudio radiológico de cadera mediante percepción táctil dirigido a estudiantes de Fisioterapia con discapacidad visual. RED Visual: Revista Especializada en Discapacidad Visual, 76, 11-23. https://doi.org/10.53094/CQIR4487. 
Murray, A. W., y Robb, J.E. (2006). The hip in cerebral palsy. Current Orthopaedics, 20(4), 286-293. (DOI: https://doi.org/10.1016/j.cuor.2006.06.011).

Vitale, M. G., y Skaggs, D. L. (2010). Displasia del desarrollo de la cadera de los seis meses a los cuatro años de edad. En: J. Albiñana Cilveti y E. Sink (coords.), Problemas de cadera en ortopedia infantil [PDF] (pp. 1-8). Madrid, España: Editorial Médica Panamericana.

María Pilar Martín Rubio. Profesora fisioterapeuta. Escuela Universitaria de Fisioterapia de la ONCE. Calle de Nuria, 42; 28034 Madrid (España). Correo electrónico: pmru@once.es.

Luis Fernando Arribas González. Especialista de Núcleos Periféricos. Escuela Universitaria de Fisioterapia de la ONCE. Calle de Nuria, 42; 28034 Madrid (España). Correo electrónico: lfag@once.es. 